\title{
Genetic Diversity of SARS-CoV-2 among Travelers Arriving in Hong Kong
}

Haogao Gu, ${ }^{1}$ Daniel K.W. Chu, ${ }^{1}$ Lydia D.J. Chang, Sammi S.Y. Cheuk, Shreya Gurung, Pavithra Krishnan, Daisy Y.M. Ng, Gigi Y.Z. Liu, Carrie K.C. Wan, Ruopeng Xie, Samuel S.M. Cheng, Benjamin J. Cowling, Dominic N.C. Tsang, Malik Peiris, Vijaykrishna Dhanasekaran, Leo L.M. Poon

We sequenced $10 \%$ of imported severe acute respiratory syndrome coronavirus 2 infections detected in travelers to Hong Kong and revealed the genomic diversity of regions of origin, including lineages not previously reported from those countries. Our results suggest that international or regional travel hubs might be useful surveillance sites to monitor sequence diversity.

$\mathrm{H}$ ong Kong uses an elimination strategy to control coronavirus disease (COVID-19) that includes stringent travel restrictions to reduce the risk of introducing severe acute respiratory syndrome coronavirus 2 (SARS-CoV-2) into local communities (1). COVID-19 testing was mandated on departure and arrival for all inbound travelers. Compulsory 14-day home quarantine was put in place for all arrivals beginning March 19, 2020. Nonresidents were banned from entry after March 25. In subsequent months, persons arriving from high-risk locations were required to quarantine in hotels; by November, all arrivals had to quarantine in hotels. On December 25, the quarantine period was extended to 21 days. Predeparture COVID-19 testing was mandated for travelers inbound from high-risk locations. Furthermore, daily health declarations were required from all quarantined travelers and respiratory samples were collected on

Author affiliations: School of Public Health, University of Hong Kong, Hong Kong, China (H. Gu, D.K.W. Chu, L.D.J. Chang, S.S.Y. Cheuk, S. Gurung, P. Krishnan, D.Y.M. Ng, G.Y.Z. Liu, C.K.C. Wan, R. Xie, S.S.M. Cheng, B.J. Cowling, M. Peiris, V. Dhanasekaran, L.L.M. Poon); Department of Health Centre for Health Protection, Government of Hong Kong Special Administrative Region, Hong Kong (D.N.C. Tsang); HKU-Pasteur Research Pole, University of Hong Kong, Hong Kong (M. Peiris, V. Dhanasekaran, L.L.M. Poon)

DOI: https://doi.org/10.3201/eid2710.211028 arrival, day 12, and day 19 (for 21-day quarantine) for reverse transcription PCR (RT-PCR) testing. As of April 25, 2021, authorities had recorded 11,731 RTPCR-positive COVID-19 cases in Hong Kong. About $20 \%(2,350)$ of the laboratory-confirmed COVID-19 cases were considered imported, detected in persons thought to have been infected outside of Hong Kong. Here, we report the analyses of $10 \%$ of these imported cases through genome sequencing.

\section{The Study}

A total of 2,192 COVID-19-positive travelers arrived in Hong Kong during January 2020-March 2021 (Appendix 1 Figure 1, https://wwwnc.cdc.gov/EID/ article/27/10/21-1028-App1.pdf). Stratifying cases by departure location (Appendix 1 Table 1) showed that 10 countries accounted for $77.8 \%$ of all imported cases during this period: United Kingdom (406), Philippines (318), India (309), Pakistan (245), Indonesia (149), United States (131), Nepal (75), Russia (40), France (33), and United Arab Emirates (25). After compulsory COVID-19 RT-PCR screening on arrival at the airport began on April 7, 2020, authorities detected 1,102 cases; 80\% (886) of case-patients were asymptomatic at the time of testing. Of 491 case-patients testing SARS-CoV-2-positive during quarantine, 69\% were asymptomatic and cases were detected a mean $( \pm$ SD) of $11.3 \pm 4.32$ days after arrival. This finding indicates that many COVID-19 cases from quarantined travelers were only identified during the first compulsory testing on day 12. These findings support Hong Kong's stringent follow-up measures for inbound travelers to prevent introduction of SARSCoV-2 into communities.

To estimate the viral sequence diversity among these imported cases, we performed next-generation

\footnotetext{
${ }^{1}$ These first authors contributed equally to this article.
} 
sequencing on $10 \%$ (221) of clinical samples collected $(2,3)$ (Appendix). We selected a greater proportion of samples (204) beginning in June 2020 when greater genetic diversity began to appear globally. The number of samples we sequenced by country of origin was proportional to all cases detected in travelers from that country $(\mathrm{R}=0.91)$.

Using the Pangolin classification system (https://github.com/hCoV-2019/pangolin), we detected 58 different SARS-CoV-2 lineages; the most common were B.1.1.7 (39), B.1.1.63 (21), B.1.36 (18), B.1 (17), and B.1.1 (17) (Figure; Appendix 1 Table 2). We detected 2 variants of concern (VOC) and 3 variants of interest (VOI; Table 1) (5). VOC B.1.1.7 (Alpha variant), which began spreading rapidly in the United Kingdom in November $2020(6,7)$, was the most common VOC (39) in our study. We first detected this lineage in a passenger arriving from the United Kingdom on December 13, 2020, and we subsequently detected it in another 38 travelers from other countries, predominantly from the Philippines and Pakistan (Table 1). This finding corresponds with data from global surveillance that indicate this lineage has been circulating over a wide geographic range beginning in December 2020. The second VOC, B.1.351 (Beta), which was first reported to circulate widely in South Africa beginning in November 2020 (8), we first detected on December 16 in an arriving passenger with a recent travel history in the United Kingdom and South Africa (1). Subsequent cases caused by this variant were detected only in March 2021 in travelers from the Philippines (5) and Bangladesh (1). All 3 of the VOI we detected were imported from the countries where they were first reported to have emerged: B.1.526 (Iota) from the United States, B.1.617 (Kappa) from India, and P.3 (Theta) from the Philippines (M.K. Annavajhala et al., unpub data, https://doi.org/10.1101/2021.02 .23.21252259; S. Cherian et al., unpub data, https:/ / doi.org/10.1101/2021.04.22.440932; F.A. Tablizo et al., unpub data, https:// doi.org/10.1101/2021.03 .03.21252812). Based on sequences detected in samples from case-patients, B.1.526 was imported on March 20, B.1.617 on March 25, and P.3 on January 21,2021 . These variants were first reported to spread rapidly in these countries during February (B1.526 and B.1.617) and March 2021 (P.3), indicating that testing arrivals from outside of Hong Kong and sequencing positive samples might enable us to capture information about variants circulating in other geographic locations.

Fifty percent of our cases were imported from 5 middle-income countries in Asia: India, Indonesia,
Nepal, Pakistan, and the Philippines (https://databank.worldbank.org/data/download/site-content/ CLASS.xls; Appendix Table 1). We wanted to compare the genomic diversity of SARS-CoV-2 imported from these countries with those reported in the GISAID database (https://www.gisaid.org). However, the Philippines, Nepal, and Pakistan had limited SARS-CoV-2 sequence information in the GISAID database (Table 2) (9). Of the 3 VOC or VOI we identified in travelers from the Philippines (Table 2), B.1.351 was not among sequences the Philippines submitted to GISAID, but the March 6-20, 2021, arrival dates of the 5 case-patients with B.1.351 suggest unreported domestic circulation of that lineage. Similarly, Nepal had reported to GISAID only 15 of the 20 viral sequences from 8 lineages we had identified. Other countries also had not previously reported several lineages we identified to GISAID, including 3 from India and 1 each from Pakistan and Indonesia. We did not analyze samples from travelers from some countries, either because they had their own extensive domestic sequencing efforts or we had few samples from these countries ( $<5$ per country).

We further compared GISAID data with our data from the Philippines, Nepal, and Pakistan. We retrieved the earliest collection date for each lineage we detected that these countries had also reported to GISAID; some of those dates were close to the first dates of arrival for case-patients with those lineages in our study. In fact, in over half of those lineages reported in both sources, we identified the lineage either before or $<1$ month after it was reported by the country (Appendix Table 3), highlighting the potential use of this method of surveillance to assess genomic diversity in regions with limited sequence information.

The emergence of VOC and VOI in different geographic locations highlights the need for global-level genomic surveillance of SARS-CoV-2 (10), but genomic sequencing information from some regions remains incomplete. Our findings suggest that

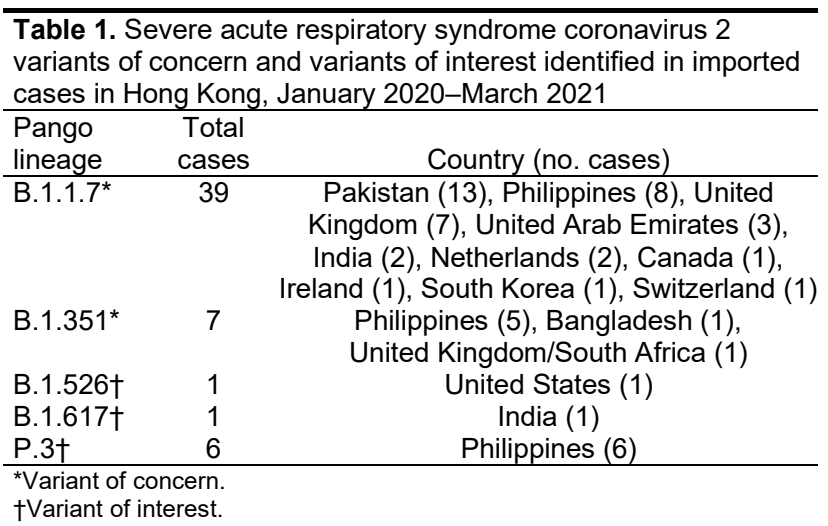


Table 2. Severe acute respiratory syndrome coronavirus 2 lineages imported from different countries in Asia into Hong Kong, January 2020-March 2021

\begin{tabular}{|c|c|c|c|}
\hline Country & $\begin{array}{l}\text { No. sequences } \\
\text { from GISAID* }\end{array}$ & $\begin{array}{l}\text { No. sequences } \\
\text { in this study }\end{array}$ & Lineages found in this study \\
\hline India & 11,435 & 32 & $\begin{array}{c}\text { B.1, B.1.1, B.1.1.1, B.1.1.306, B.1.1.7, B.1.210, B.1.36, B.1.36.18, B.1.36.29, } \\
\text { B.1.36.36, † B.1.36.8, B.1.369, B.1.562, †.1.589, † B.1.617 }\end{array}$ \\
\hline Indonesia & 1,170 & 18 & B.1.1, B.1.1.272,† B.1.1.398, B.1.36.19, B.1.459, B.1.468, B.1.470 \\
\hline Philippines & 188 & 47 & B.1.1, B.1.1.263, B.1.1.63, B.1.1.7, B.1.351,† B.6, P.3 \\
\hline Pakistan & 136 & 21 & A.21,† B.1, B.1.1.1, B.1.1.7, B.1.36, B.1.471 \\
\hline Nepal & 15 & 20 & B.1.1, B.1.1.214,† B.1.1.216, B.1.36, B.1.36.18,† B.1.36.22,† B.1.36.27,† B.1.468† \\
\hline
\end{tabular}

travel hubs such as Hong Kong can be used as surveillance sites to identify infected travelers from regions with widespread circulation of lineages of interest. Such indirect surveillance might provide useful data to partially reveal virus diversity in countries with limited sequence information, leading to better preparedness for and response to newly emerging SARS-CoV-2 variants. However, findings from these indirect analyses are likely to be only partial and skewed by the level of passenger traffic to destination countries from various points of departure. Also, the extent of different virus lineages circulating in a country of departure may have affected our observations; lineages that circulate at a low level in a country of interest might be missed by our current strategy. Optimizing this approach, such as by directing sequencing efforts toward travelers departing from targeted countries or regions rather than at the points of arrival, might help overcome those limitations.

\section{Acknowledgments}

We gratefully acknowledge the efforts of the staff of the laboratories responsible for obtaining the specimens and the laboratories receiving submissions where genomic data were generated and shared via GISAID (Appendix 2, https://wwwnc.cdc.gov/EID/article/27/10/21-1028App2.xlsx). We acknowledge the technical support provided by colleagues from the Centre for PanorOmic Sciences of the University of Hong Kong.

This work was supported by grants from the National Institute of Allergy and Infectious Diseases (U01AI151810, HHSN272201400006C), the Health and Medical Research Fund (COVID190205), and the Collaborative Research Fund, RGC (C7123-20G). We also acknowledge the Centre for Health Protection of the Department of Health for providing epidemiologic data for the study.

Virus sequences reported in this study are available from GISAID. The epidemiological data for these imported cases can be accessed in a public database (https:// data.gov.hk/ en-data/dataset/hk-dh-chpsebcddr-novel-infectious-agent).

\section{About the Author}

Dr. Gu is a postdoctoral fellow at the University of Hong Kong. His interests focus on bioinformatics and virus evolution.

\section{References}

1. Xue W, Lam C, Yeung HH, Wong CS, Chan VLY, Wong YS. Travel restrictions in the rising COVID-19 pandemic. Hong Kong Med J. 2020;26:255-7.

2. Choi EM, Chu DKW, Cheng PKC, Tsang DNC, Peiris M, Bausch DG, et al. In-flight transmission of SARS-CoV-2. Emerg Infect Dis. 2020;26:2713-6. https:/ / doi.org/10.3201/ eid2611.203254

3. Sit THC, Brackman CJ, Ip SM, Tam KWS, Law PYT, To EMW, et al. Infection of dogs with SARS-CoV-2. Nature. 2020; 586:776-8. https://doi.org/10.1038/s41586-020-2334-5

4. Rambaut A, Holmes EC, O’Toole Á, Hill V, McCrone JT, Ruis C, et al. A dynamic nomenclature proposal for SARS-CoV-2 lineages to assist genomic epidemiology. Nat Microbiol. 2020;5:1403-7. https://doi.org/10.1038/ s41564-020-0770-5

5. CDC. SARS-CoV-2 Variant classifications and definitions.2021 [cited 2021 Apr 30] https:/ / www.cdc.gov/ coronavirus/2019-ncov/cases-updates/variant-surveillance/ variant-info.html

6. Leung K, Shum MH, Leung GM, Lam TT, Wu JT. Early transmissibility assessment of the N501Y mutant strains of SARS-CoV-2 in the United Kingdom, October to November 2020. Euro Surveill. 2021;26:2002106. https:/ / doi.org/10.2807/1560-7917.ES.2020.26.1.2002106

7. Davies NG, Abbott S, Barnard RC, Jarvis CI, Kucharski AJ, Munday JD, et al.; CMMID COVID-19 Working Group; COVID-19 Genomics UK (COG-UK) Consortium. Estimated transmissibility and impact of SARS-CoV-2 lineage B.1.1.7 in England. Science. 2021;372:eabg3055. https://doi.org/10.1126/science.abg3055

8. Tegally H, Wilkinson E, Giovanetti M, Iranzadeh A, Fonseca V, Giandhari J, et al. Detection of a SARS-CoV-2 variant of concern in South Africa. Nature. 2021;592:438-43. https:/ / doi.org/10.1038/s41586-021-03402-9

9. Elbe S, Buckland-Merrett G. Data, disease and diplomacy: GISAID's innovative contribution to global health. Glob Chall. 2017;1:33-46. https://doi.org/10.1002/gch2.1018

10. The Lancet. Genomic sequencing in pandemics. Lancet. 2021;397:445. https:// doi.org/10.1016/S0140-6736(21)00257-9

Address for correspondence: Leo Poon, School of Public Health, University of Hong Kong, 21 Sassoon Rd, Pokfulam, Hong Kong; email: 1lmpoon@hku.hk 